\title{
A new subspace-based approach to identify nonlinear mechanical structures in the frequency domain
}

\author{
J.P. Noël, G. Kerschen \\ Space Structures and Systems Laboratory (S3L) \\ Department of Aerospace and Mechanical Engineering \\ University of Liège, Belgium \\ jp.noel,g.kerschen@ulg.ac.be
}

\begin{abstract}
:
This paper introduces a new frequency-domain subspace-based method for the identification of nonlinear mechanical systems. The technique exploits frequency data and interprets nonlinearities as feedback forces exciting the underlying linear system. It is demonstrated using two academic examples, a Duffing oscillator and a five degree-of-freedom system comprising two nonlinearities.
\end{abstract}

Keywords: Nonlinear system identification, frequency signal analysis, subspace methods.

\section{INTRODUCTION}

Subspace techniques are powerful tools to identify linear time-invariant systems. Many alternative formulations were proposed in the technical literature, both in the time (Van Overschee and De Moor [1996a]) and in the frequency (McKelvey et al. [1996]) domains. Nonlinear system identification is much more challenging, in particular in structural dynamics (Kerschen et al. [2006]). However, recent advances (Lacy and Bernstein [2005], Marchesiello and Garibaldi [2008]) have shown that subspace-based algorithms in the time domain can accurately handle nonlinear data. This work proposes to consider frequency data in such algorithms and results in the introduction of a new nonlinear system identification method dedicated to mechanical structures, referred to as the Frequencydomain Nonlinear Subspace Identification (FNSI) method. Two numerical examples are studied to demonstrate the accuracy and the efficiency of the proposed strategy. The selection of informative frequency regions will also be analysed.

\section{PROBLEM STATEMENT}

The present paper addresses the identification of nonlinear mechanical systems whose nonlinearities are supposed to be localised and for which there exists an underlying linear regime of vibration. The amplitude, the direction and the frequency content of the excitation determine in which regime the structure behaves. The vibrations of such nonlinear systems are governed by the time-continuous model

$$
M \ddot{q}(t)+C \dot{q}(t)+K q(t)+f(q(t), \dot{q}(t))=p(t)
$$

where $M, C, K \in \mathbb{R}^{r \times r}$ are the mass, damping and stiffness matrices, respectively, $q(t)$ and $p(t) \in \mathbb{R}^{r}$ are the generalised displacement and external force vectors, $f(t) \in \mathbb{R}^{r}$ is the nonlinear restoring force vector and $r$ is the number of degrees of freedom (DOFs) of the structure obtained after spatial discretisation.

In the frequency domain, this model becomes

$$
B(\omega) Q(\omega)+F(\omega)=P(\omega)
$$

where $Q(\omega), F(\omega)$ and $P(\omega)$ are the Fourier transforms of $q(t), f(t)$ and $p(t)$, respectively, and $B(\omega)=$ $\left(-\omega^{2} M+i \omega C+K\right)$ is the linear dynamic stiffness matrix.

The effects of the $s$ lumped nonlinearities are modelled using the summation

$$
f(q(t), \dot{q}(t))=\sum_{j=1}^{s} \mu_{j} b_{j} g_{j}(q(t), \dot{q}(t)) .
$$

Each term contains information about the strength of the nonlinearity $\mu_{j}$, its location $b_{j} \in \mathbb{R}^{r}$ and its functional form $g_{j}(t)$. Though it is a key problem, the selection of the nonlinear basis functions $g_{j}(t)$ is not treated in this work for the sake of conciseness and simplicity. However, the technical literature proposes many methods for their determination. For instance, the restoring force surface method (Noël et al. [2012]) can provide a useful visualisation of the nonlinearity and help in the choice of an appropriate functional form. Other approaches can be mentioned as the use of series expansions of the nonlinear restoring force $f(t)$ or a local modelling through low-order splines.

In this paper, we therefore focus on the estimation of the nonlinear parameters $\mu_{j}$ based on the knowledge of $b_{j}$ and $g_{j}(t)$. We also seek a description of the underlying linear dynamics of the structure through the estimation of $B(\omega)$ or its inverse, the frequency response function (FRF) matrix $H(\omega)=B^{-1}(\omega)$. 


\section{IDENTIFICATION METHODOLOGY}

The FNSI technique is a five-step methodology. They will be successively described in the present section. The main contributions of this work lie in the second and fourth steps of the identification process.

\subsection{Step 1: Joint collection of excitations and nonlinearities as input data}

An appealing approach for interpreting the dynamics governed by Equation (1) consists in moving the nonlinear term to the right-hand side and viewing nonlinear forces as external forces applied to the underlying linear structure:

$$
M \ddot{q}(t)+C \dot{q}(t)+K q(t)=p(t)-f(t) .
$$

Doing so, the internal forces that are nonlinear functions of the outputs act as a feedback to the linear open-loop system. This peculiar feedback form allows the transition to an equivalent linear system identification problem (Lacy and Bernstein [2005]). Moreover, we opt for a discretetime state-space modelling for numerical conditioning reasons (Van Overschee and De Moor [1996b]).

Thus, our objective is to fit to observations a state-space model of the form

$$
\left\{\begin{aligned}
x(t+1) & =A x(t)+B u(y, t) \\
y(t) & =C x(t)+D u(y, t)
\end{aligned}\right.
$$

where $A \in \mathbb{R}^{n \times n}$ prescribes the linear dynamics of the system, $B \in \mathbb{R}^{n \times m}$ contains the parameters $\mu_{j}$ that are eventually sought, $C \in \mathbb{R}^{l \times n}$ is the output matrix, $D \in \mathbb{R}^{l \times m}$ is the direct feed-through matrix, $u(y, t) \in$ $\mathbb{R}^{m}$ concatenates the excitation signals and the nonlinear describing functions $g_{j}(t), x(t)=\left(\begin{array}{ll}q & \dot{q}\end{array}\right)^{T} \in \mathbb{R}^{n}$ is the state vector, $y(t) \in \mathbb{R}^{l}$ is the measured output vector and $n=2 r$.

\subsection{Step 2: Formulation in the frequency domain}

Tackling system identification problems in the frequency domain is an attractive and versatile alternative (Pintelon and Schoukens [2001]). Furthermore, experimental data are often recorded as FRFs, power spectral densities or simply spectra. It is also worth pointing out that frequency-domain subspace algorithms proved successful in linear identification problems (Pintelon [2002]). This motivates us to approach nonlinear mechanical structures by using subspace methods formulated in the frequency domain. To this end, Equations (5) are recast by use of the Discrete Fourier Transform (DFT).

The DFT $V(k)$ of a time signal $v(t)$ is given by ${ }^{1}$

$$
V(k)=\frac{1}{\sqrt{M}} \sum_{t=0}^{M-1} v(t) e^{-j 2 \pi k t / M}
$$

where $M$ is the number of recorded time samples of $v, k$ is the frequency line and $j$ is the imaginary unit.

\footnotetext{
1 Provided that $v(t)$ is periodic and observed over an integer number of periods in steady-state conditions.
}

Equations (5) thus write

$$
\left\{\begin{aligned}
z_{k} X(k) & =A X(k)+B U(k) \\
Y(k) & =C X(k)+D U(k)
\end{aligned}\right.
$$

where $z_{k}$ is the Z-transform variable

$$
z_{k}=e^{j 2 \pi k / M}
$$

and $X(k), U(k)$ and $Y(k)$ are the DFTs of $x(t), u(y, t)$ and $y(t)$, respectively.

Equations (6) is commonly recast into matrix form in the subspace literature $^{2}$ :

$$
Y_{i}=\Gamma_{i} X+H_{i} U_{i}
$$

where $Y_{i}, U_{i}$ and $X$ gather entire spectra, $\Gamma_{i}$ and $H_{i}$ contain the state matrices only and the index $i$ indicates the number of block rows of a matrix. The extended obversability matrix $\Gamma_{i}$ plays a major role in subspace theory as underlined in Step 3 and Equation (7) will be exploited in Step 4.

\subsection{Step 3: Estimation of $\Gamma_{i}, A$ and $C$}

The purpose of subspace algorithms is the computation of the extended observability matrix using geometrical projections of input-output data. This computation starts with the cancellation of the input term in Equation (7) by means of orthogonal projection (Van Overschee and De Moor [1996a]). Secondly, estimates of $\Gamma_{i}$ and of the system order $n$ are obtained through a truncated Singular Value Decomposition (SVD). To detail this procedure (see (Pintelon [2002])) and to examine its theoretical foundations (see (Lacy and Bernstein [2005])) are beyond the scope of this paper.

From the knowledge of $\Gamma_{i}$, further stages then consist in finding the best way to estimate the state matrices. In frequency-domain algorithms, $A$ and $C$ are efficiently computed from the so-called shift property of $\Gamma_{i}$ (McKelvey et al. [1996]). We also follow this way in our methodology. An effective computation of $B$ and $D$ is more delicate and no satisfactory solution can be found in the literature.

\subsection{Step 4: Proposed estimation of $B$ and D}

The core of the demonstration is inspired by (Van Overschee and De Moor [1996a], pp. 118-127) although several major adaptations are proposed to meet our frequencydomain formulation.

First, we substitute in Equations (6) the state spectrum $X$ and its frequency derivative $X \zeta$ by using the outputstate-input Equation (7) and its transformed version

$$
Y_{i} \zeta=\Gamma_{i} X \zeta+H_{i} U_{i} \zeta
$$

where $\zeta=\operatorname{diag}\left[\begin{array}{llll}z_{1} & z_{2} & \ldots & z_{N}\end{array}\right]$ considering $N$ (non-necessarily equidistant) frequency lines. We secondly observe that

$$
\left\{\begin{array} { c } 
{ Y _ { i } = \underline { Y } _ { i + 1 } } \\
{ U _ { i } = \underline { U } _ { i + 1 } }
\end{array} \text { and } \left\{\begin{array}{c}
Y_{i} \zeta=\bar{Y}_{i+1} \\
U_{i} \zeta=\bar{U}_{i+1}
\end{array}\right.\right.
$$

where the operators $\underline{Z}$ and $\bar{Z}$ remove from their argument $\mathrm{Z}$ the last and first block rows, respectively. These shift

\footnotetext{
${ }^{2}$ For more details, we refer the reader to (Van Overschee and De Moor [1996b]).
} 
properties can be realised by simply considering the data matrix structure presented, for instance, in (Van Overschee and De Moor [1996b]). We consequently eliminate $X$ and $X \zeta$ by the relations

$$
\left\{\begin{aligned}
X & =\Gamma_{i}^{\dagger}\left(\underline{Y}_{i+1}-H_{i} \underline{U}_{i+1}\right) \\
X \zeta & =\Gamma_{i}^{\dagger}\left(\bar{Y}_{i+1}-H_{i} \bar{U}_{i+1}\right)
\end{aligned}\right.
$$

where $\dagger$ denotes the inverse of a non-square matrix.

For the sake of convenience, identity and zero matrices are preferred to under- and overline operators as

$$
\left\{\begin{aligned}
X= & \Gamma_{i}^{\dagger}\left(\mathrm{I}^{[l i]}-0^{[l i \times l]}\right) Y_{i+1} \\
& -\Gamma_{i}^{\dagger} H_{i}\left(\mathrm{I}^{[m i]}-0^{[m i \times m]}\right) U_{i+1} \\
X \zeta= & \Gamma_{i}^{\dagger}\left(0^{[l i \times l]}-\mathrm{I}^{[l i]}\right) Y_{i+1} \\
& -\Gamma_{i}^{\dagger} H_{i}\left(0^{[m i \times m]}-\mathrm{I}^{[m i]}\right) U_{i+1} .
\end{aligned}\right.
$$

Some simple manipulations lead to the final writing of the state-space equations

$$
\begin{gathered}
\left(\frac{\Gamma_{i}^{\dagger}\left(0^{[l i \times l]}-\mathrm{I}^{[l i]}\right) Y_{i+1}}{Y}\right)= \\
\left(\frac{A}{C}\right) \Gamma_{i}^{\dagger}\left(\mathrm{I}^{[l i]}-0^{[l i \times l]}\right) Y_{i+1}+\mathcal{K} U_{i+1}
\end{gathered}
$$

where

$$
\mathcal{K}=\left(\frac{\left(B-\Gamma_{i}^{\dagger} H_{i}\right)-\left(A \Gamma_{i}^{\dagger} H_{i}-0^{[m i \times m]}\right)}{\left(D-0^{[l \times m i]}\right)-\left(C \Gamma_{i}^{\dagger} H_{i}-0^{[m i \times m]}\right)}\right) .
$$

Two features of Equation (12) must be emphasised. First, the matrix $\mathcal{K}$, which exclusively contains the state matrices, is the single unknown of the problem. The new quantities $\mathcal{P}$ and $\mathcal{Q}$ are thus introduced as

$$
\begin{aligned}
\mathcal{P}= & \left(\frac{\Gamma_{i}^{\dagger}\left(0^{[l i \times l]}-\mathrm{I}^{[l i]}\right) Y_{i+1}}{Y}\right)- \\
& \left(\frac{A}{C}\right) \Gamma_{i}^{\dagger}\left(\mathrm{I}^{[l i]}-0^{[l i \times l]}\right) Y_{i+1}
\end{aligned}
$$

and

$$
\mathcal{Q}=U_{i+1}
$$

Therefore, $B$ and $D$ can be computed as the solution of the least-squares problem

$$
B, D=\arg \min _{B, D}\|\mathcal{P}-\mathcal{K}(B, D) \mathcal{Q}\|^{2} .
$$

Secondly, $\mathcal{K}$ is linear in $B$ and $D$. This causes the aforementioned optimisation problem to be convex. Despite this appealing property, we pursue another way to solve the problem. Indeed, for efficiency purposes, Equation (13) is eventually rewritten as an explicit linear combination of $B$ and $D$ and solved in least-squares sense.

\subsection{Step 5: Conversion of the system model into the nonlinearity model}

The final aim of our approach is the computation of the coefficients $\mu_{j}$ and the FRF matrix $H(\omega)$ and not only the fitting of Equations (6) to experimental data. To that purpose, we strictly follow the idea of (Marchesiello and Garibaldi [2008]). In brief, an invariant combination (with respect to a similarity transformation) of the estimated state matrices is built (termed extended frequency response function) from which the coefficients and the FRFs can be easily extracted. The main feature of this computation is that it provides complex and frequency-dependent coefficients. To ensure their physical meaning, a reliable identification scheme should minimise their imaginary parts and their frequency variations. We will exploit these quality criteria in the next section.

\section{NUMERICAL EXAMPLES}

We demonstrate the capabilities of our new identification algorithm on two numerical examples: a Duffing oscillator and a five DOF system with two nonlinearities taken from (Richards and Singh [1998]). This latter paper introduces the Conditioned Reverse Path (CRP) method. It will serve as a reference to assess the accuracy and the time efficiency of the FNSI method in the first example. The excellent identification capabilities of the CRP method were already demonstrated in the technical literature (Kerschen et al. [2003]). However, it shows difficulties in tackling structures with multiple nonlinearities. In the MultiDegree-Of-Freedom (MDOF) example, the FNSI results will prove to overcome this limitation.

\subsection{Duffing oscillator}

The vibrations of a Duffing oscillator are governed by the equation

$$
M \ddot{q}(t)+C \dot{q}(t)+K q(t)+\mu q^{3}(t)=p(t) .
$$

The linear and nonlinear parameters of the system are listed in Table 1. It was simulated using a nonlinear Newmark time integration scheme (sampling frequency of $2,500 \mathrm{~Hz}$ ). A periodic noise excitation was considered ${ }^{3}$. To that purpose, a single band-limited $(0-100 \mathrm{~Hz})$ random signal (12,500 points) was repeated 40 times. Its Root Mean Square (RMS) value was set to 100 N. As a general practice, transients were removed prior to processing data.

Only 17,100 out of 450,000 frequency lines will be considered spanning from 5 to $100 \mathrm{~Hz}$. Low frequency and out-of-input-band components are thus rejected from our analysis since the information content they convey is less valuable, except for harmonic regions. In comparison with time-domain data, the full time series would be processed leading to an increased computational burden. The possibility to focus on highly informative frequency regions is the central property of the FNSI method.

\begin{tabular}{c|c} 
Mass $M$ & $2(\mathrm{~kg})$ \\
Damping $C$ & $5(\mathrm{Ns} / \mathrm{m})$ \\
Stiffness $K$ & $100(\mathrm{kN} / \mathrm{m})$ \\
Cubic stiffness $\mu$ & $10\left(\mathrm{MN} / \mathrm{m}^{3}\right)$ \\
Damped frequency & $35.59(\mathrm{~Hz})$ \\
Damping percentage & $0.6(\%)$ \\
\hline
\end{tabular}

Table 1 . Parameters of the Duffing oscillator. 3 A formal definition of a periodic noise can be found in (Pintelon
and Schoukens [2001], p. 73). 
Figure 1 displays the complex and frequency-dependent estimation of the cubic stiffness. Its real part has no visible dependence upon frequency and its imaginary part is 5 orders of magnitude smaller. These are proofs of an excellent quality estimate, as detailed in Table 2 (first row). We stress the high computational efficiency of the method. Less than a second is indeed enough to retrieve the exact value of the nonlinear parameter.

Figure 2 and Table 2 (second row) show the comparison with the CRP method. The FNSI method clearly yields a much more stable coefficient with a considerably reduced effort. Besides, we show in Figure 3 that the reconstruction of the underlying linear FRF of the system from nonlinear data is also accurately handled.

As mentioned above, the main advantage of a frequencydomain formulation is that a natural selection of information can be made according to frequencies, as illustrated in Table 2 (third row). By focusing on a high signal-to-noise ratio band of the spectra between 29 and $42 \mathrm{~Hz}$ (i.e. the neighbourhood of the resonance peak), the identification can be improved and is faster (2,340 frequency lines processed).
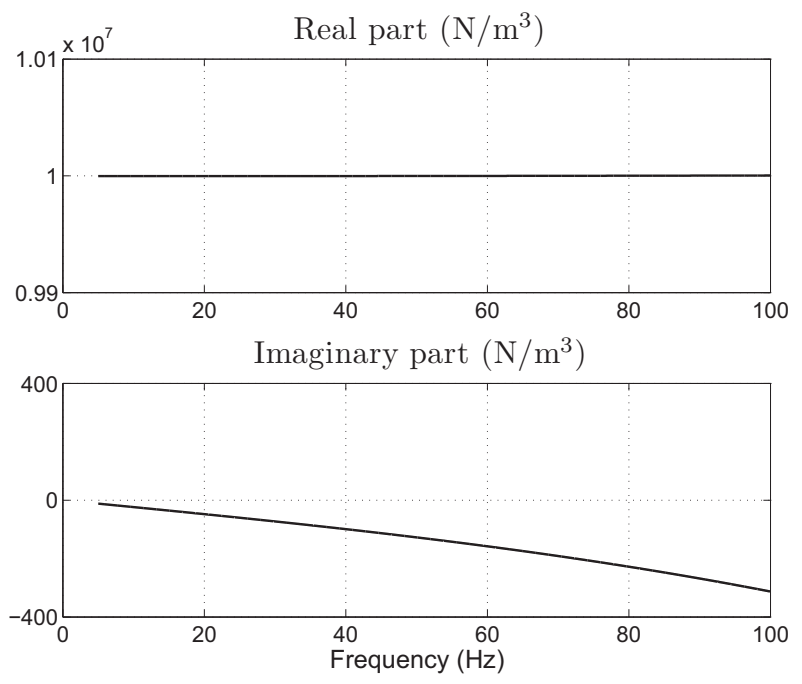

Fig. 1. FNSI estimation of $\mu$ over the $5-100 \mathrm{~Hz}$ band. The vertical axis of the real part is chosen such that $1 \%$ error bounds are visible.

\begin{tabular}{c|c|c|c|c} 
& Real part & Error $(\%)$ & Real/Imag. & Time $(s)$ \\
\hline FNSI & & & & \\
$(5-100 \mathrm{~Hz})$ & 10.00 & $0.810^{-3}$ & $69.510^{3}$ & 0.3 \\
CRP & 9.98 & 0.2 & 203.1 & 5.4 \\
FNSI & & & & \\
$(29-42 \mathrm{~Hz})$ & 10.00 & $0.510^{-3}$ & $1.010^{6}$ & 0.1 \\
\hline Table & $2 . \quad$ Estimation of the coefficient & $\mu$
\end{tabular}

$$
\left(M N / m^{3}\right) \text {. }
$$

\subsection{MDOF system with two nonlinearities}

We now address the five DOF structure displayed in Figure 4 . The linear stiffnesses $k_{i}$ of the system are chosen equal to $50 \mathrm{kN} / \mathrm{m}$ expect $k_{6}=35 \mathrm{kN} / \mathrm{m}$. The masses $m_{i}$ and the dampings $c_{i}$ are all equal to $1 \mathrm{~kg}$ and $10 \mathrm{Ns} / \mathrm{m}$,
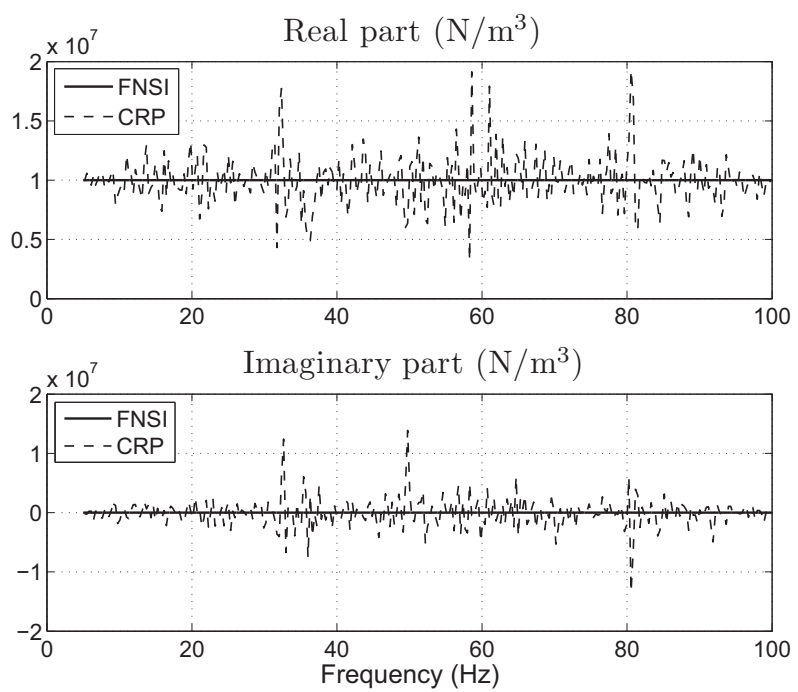

Fig. 2. Comparison between the FNSI and CRP results. The latter method provides a highly oscillating coefficient.

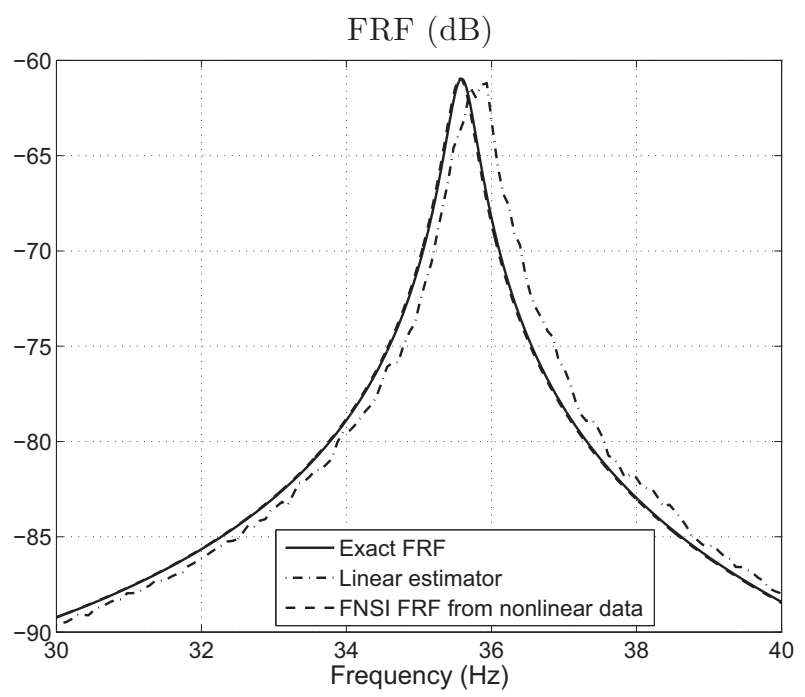

Fig. 3. FNSI reconstruction of the linear FRF. The relative errors (in \%) on the identified damped frequency (0.001) and damping percentage (0) are negligible.

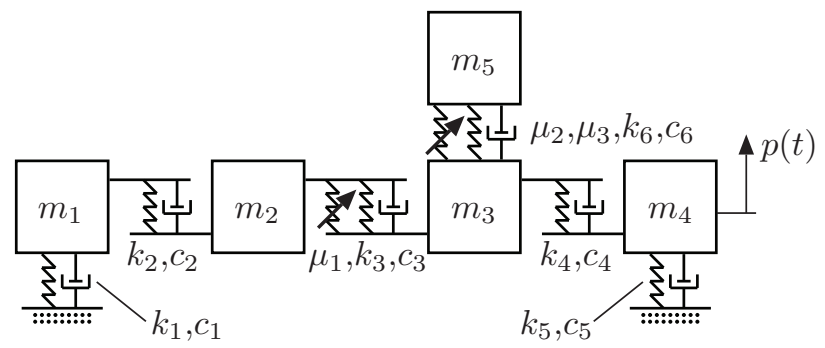

Fig. 4. Schematic view of the MDOF example.

respectively. The linear properties of the structure are given in Table 3 .

A cubic nonlinearity connects the masses $m_{2}$ and $m_{3}$ while an asymmetric quadratic-fifth order nonlinearity connects 
$m_{3}$ and $m_{5}$. The exact values of their coefficients are listed in Table 4. A Newmark time integration scheme was used considering a sampling frequency of 5,000 $\mathrm{Hz}$.

A single force $p(t)$ was finally applied on mass 4. A periodic band-limited (0-100 $\mathrm{Hz})$ noise was considered again consisting of a single random signal (10,000 points) repeated 40 times. A RMS value of $500 N$ was selected.

\begin{tabular}{c|c|c|c} 
Mode & $\begin{array}{c}\text { Damped } \\
\text { freq. }(\mathrm{Hz})\end{array}$ & $\begin{array}{c}\text { Damping } \\
\text { perc. }(\%)\end{array}$ & Linear mode shape \\
\hline 1 & 17.34 & 1.2 & $0.22 ; 0.39 ; 0.47 ; 0.27 ; 0.71$ \\
2 & 33.09 & 2.5 & $-0.50 ;-0.57 ;-0.15 ;-0.13 ; 0.62$ \\
3 & 44.42 & 3.0 & $0.42 ; 0.19 ;-0.34 ;-0.77 ; 0.28$ \\
4 & 58.82 & 3.8 & $-0.68 ; 0.50 ; 0.31 ;-0.43 ;-0.11$ \\
5 & 71.25 & 4.7 & $-0.24 ; 0.49 ;-0.74 ; 0.37 ; 0.16$ \\
\hline
\end{tabular}

Table 3. Linear dynamic properties of the system.

$$
\begin{array}{c|c|c}
\mu_{1}\left(M N / m^{3}\right) & \mu_{2}\left(k N / m^{2}\right) & \mu_{3}\left(G N / m^{5}\right) \\
\hline 500 & -500 & 10
\end{array}
$$

Table 4. Nonlinear coefficients to be estimated.

They are of different orders of magnitude and different signs.

The identification of the system is carried out considering spectra from 25 to $85 \mathrm{~Hz}$ (4,320 frequency lines). Indeed, we will qualitatively show that the first mode of the structure (around $17 \mathrm{~Hz}$ ) does not involve nonlinear behaviour because of the corresponding in-phase motion of the five DOFs. This demonstrates again the attractive flexibility of a frequency-domain formulation. Such a discrimination between modes would be much more complicated in the time domain. On the other hand, the estimated coefficients will be displayed over a wider band, from 5 to $100 \mathrm{~Hz}$. In this manner, the difference between the region over which the state-space model (Equations (6)) is identified and its interval of exploitation to assess the coefficients $\mu_{1}, \mu_{2}$ and $\mu_{3}$ (see Step 5) is highlighted.

The inspection of the Singular Value Plot (SVP) depicted in Figure 5 clearly suggests a model order $n$ equal to 10. In this work, we will not analyse the robustness of the identification versus a misestimation of the order. However, the easy interpretation of the result in Figure 5 can be extended as a recurrent property of the FNSI method and thus a sign of robustness.

The reconstruction from nonlinear data of the FRF $H_{54}(\omega)$ is presented in Figure 6 . The improvement with respect to the use of a classical H2 estimator is obvious. This graph also proves that the nonlinearities hardly corrupt the first mode around $17 \mathrm{~Hz}$ as already stated.

The estimates of $\mu_{1}, \mu_{2}$ and $\mu_{3}$ are finally displayed in Figure 7 (a), (b) and (c), respectively. In general, their are of excellent quality. Their real parts are almost constant whereas their imaginary parts remain 5 orders of magnitude lower (see Table 5). Once again, we stress the rapidity of the algorithm to yield a highly predictive model (0.8 second).

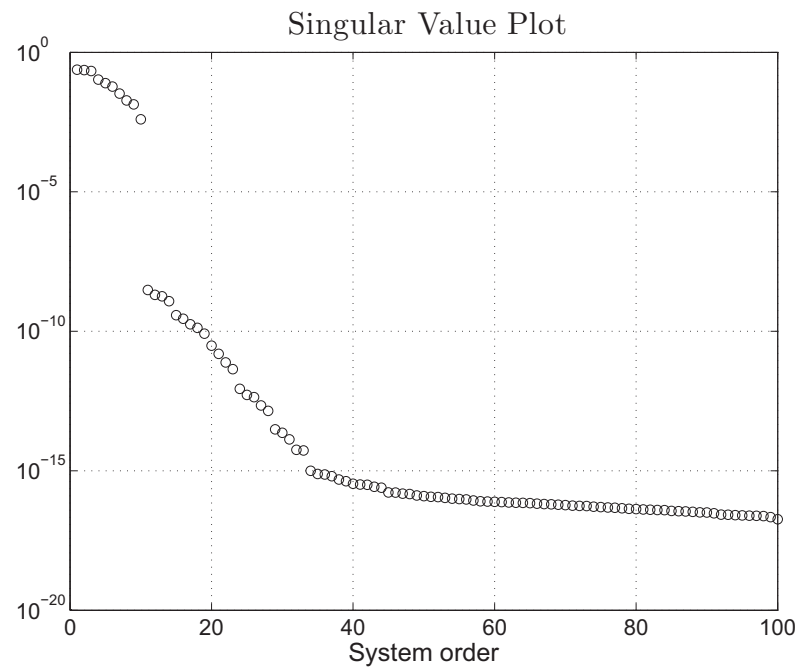

Fig. 5. Estimation of the system order $n$ from a SVP.

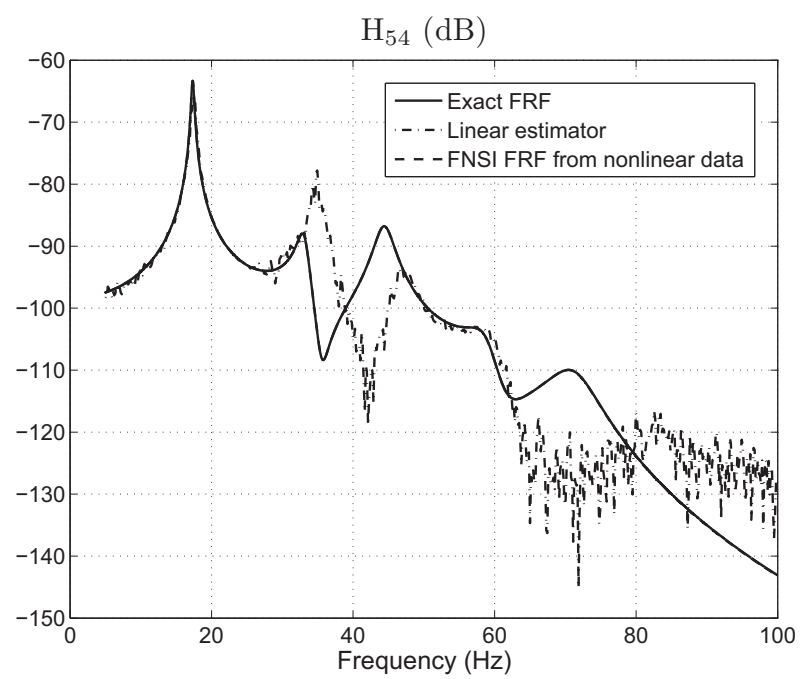

Fig. 6. The hardening behaviour of the system can be observed except on the first mode.

\begin{tabular}{c|c|c|c} 
& Real part & Error $(\%)$ & Real/Imaginary \\
\hline$\mu_{1}\left(M N / m^{3}\right)$ & 500.00 & $2.110^{-3}$ & $2.510^{5}$ \\
$\mu_{2}\left(k N / m^{2}\right)$ & -500.00 & $0.410^{-3}$ & $1.610^{5}$ \\
$\mu_{3}\left(G N / m^{5}\right)$ & 10.00 & $3.010^{-3}$ & $4.610^{5}$ \\
\hline
\end{tabular}

Table 5. Summary of the FNSI identification results.

\section{CONCLUSION}

The objective of this paper is to introduce a frequencydomain nonlinear subspace algorithm. The presented identification results are promising. In both examples discussed herein, the nonlinear coefficients were accurately and rapidly estimated. Moreover, the possibility to select frequencies considering informative bands was also demonstrated.

However, further investigations are needed to assess the capabilities of the method. First of all, the influence of measurement and process noise should be carefully analysed. A robust estimation of the system order is also 

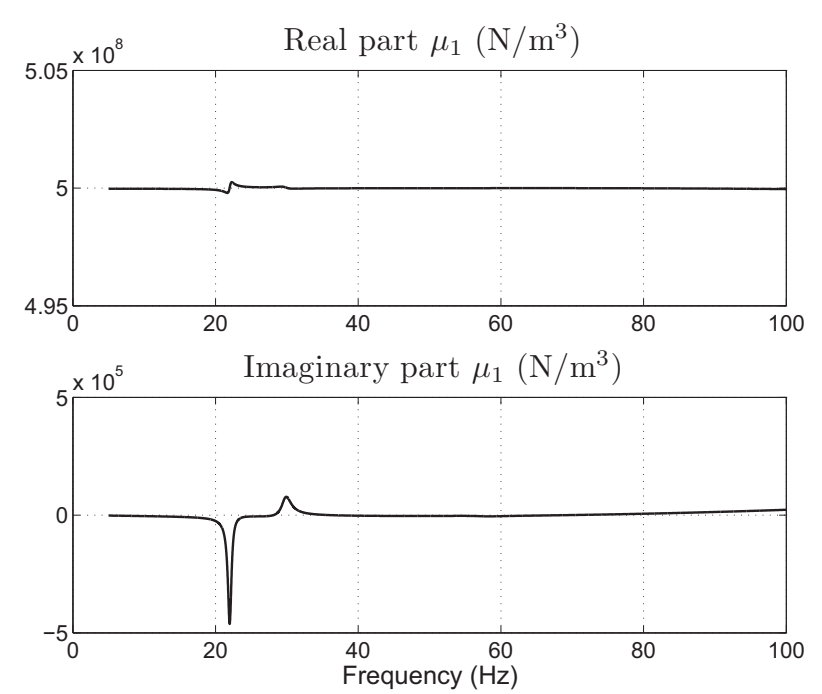

(a)
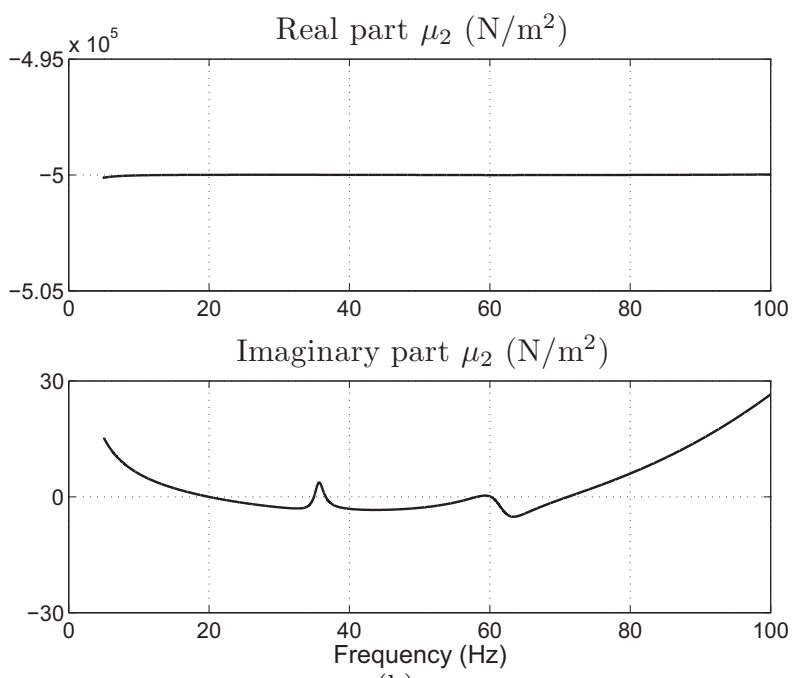

(b)
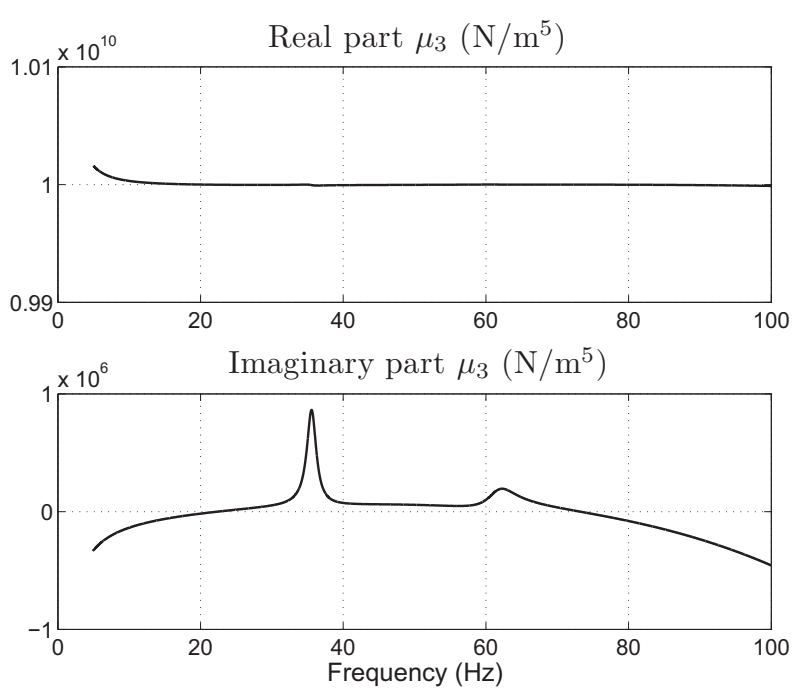

(c)

Fig. 7. Simultaneous FNSI estimation of the three nonlinear parameters. The real parts are displayed within $1 \%$-error bounds again. needed. Thirdly, quantitative means of assessing frequency regions of interest would be valuable.

Undergoing research focuses on the application of the FNSI method to real-life structures. The possibility to exploit it for non-parametric nonlinearity identification is also envisaged.

\section{ACKNOWLEDGEMENTS}

The author J.P. Noël would like to acknowledge the Belgian National Fund for Scientific Research (FRIA fellowship) for its financial support.

\section{REFERENCES}

G. Kerschen, V. Lenaerts, and J.C. Golinval. Identification of a continuous structure with a geometrical non-linearity. Part I: Conditioned reverse path method. Journal of Sound and Vibration, 2003.

G. Kerschen, K. Worden, A. F. Vakakis, and J.C. Golinval. Past, present and future of nonlinear system identification in structural dynamics. Mechanical Systems and Signal Processing, 2006.

S. L. Lacy and D. S. Bernstein. Subspace identification for non-linear systems with measured-input non-linearities. International Journal of Control, 2005.

S. Marchesiello and L. Garibaldi. A time domain approach for identifying nonlinear vibrating structures by subspace methods. Mechanical Systems and Signal Processing, 2008.

T. McKelvey, H. Akçay, and L. Ljung. Subspace-based multivariable system identification from frequency response data. IEEE Transactions on Automatic Control, 1996.

J.P. Noël, L. Renson, G. Kerschen, and A. Newerla. Experimental identification of a nonlinear space structure. In Proceedings of the 12th european conference on spacecraft structures, materials and environmental testing, 2012.

R. Pintelon. Frequency-domain subspace system identification using non-parametric noise models. Automatica, 2002.

R. Pintelon and J. Schoukens. System Identification: A Frequency Domain Approach. IEEE Press, 2001.

C. M. Richards and R. Singh. Identification of multidegree-of-freedom non-linear systems under random excitations by the "reverse path" spectral method. Journal of Sound and Vibration, 1998.

P. Van Overschee and B. De Moor. Subspace Identification for Linear Systems. Kluwer Academic Publishers, 1996a.

P. Van Overschee and B. De Moor. Continuous-time frequency domain subspace system identification. Signal Processing, 1996b. 